\title{
DEL SILENCIO A LA PALABRA. LA EXPERIENCIA DE LAS LIDERESAS INDÍGENAS DE XOCHISTLAHUACA, GUERRERO
}

\author{
IRMA GUADALUPE AGUIRRE PÉREZ \\ Grupo Interdisciplinario sobre Mujer, Trabajo y Pobreza, A.C. (México)
}

El presente artículo tiene como objetivo reflexionar sobre las condiciones y características de la participación social y política de las mujeres indígenas, específicamente sobre el caso de las mujeres que viven en la comunidad indígena de Xochistlahuaca, ubicada en el estado de Guerrero en México. Estas mujeres son parte del grupo étnico denominado amuzgos, quienes habitan una región en este estado que, junto con Oaxaca y Chiapas, presenta los más altos índices de marginación, pobreza y pobreza extrema del país.

La participación política de las mujeres en Xochistlahuaca remite a un proceso que sin duda está ligado al contexto histórico. La trangresión puede ser el adjetivo que mejor exponga lo que conlleva ser mujer, indígena y lideresa. Por ello vale una breve información del estado y los procesos políticos que han repercutido en esta región.

\section{EL ESTADO DE GUERRERO}

Ser mujer indígena en Guerrero significa resistir en extremo, y más que los hombres incluso, por todas las condiciones adversas que caracterizan al estado; por ello es importante ubicar el marco socioeconómico, histórico, cultural y geográfico en el cual se está "visibilizando» a las mujeres indígenas.

Los recursos naturales con que cuenta Guerrero ${ }^{2}$ hasta ahora han sido explotados de manera inequitativa, por lo cual los pueblos y sus habitantes han

1. Concepto planteado y citado por Massolo, Alejandra: «Introducción. Gobierno municipal y mujeres: un encuentro posible», en Dalia Barrera Bassols y Alejandra Massolo (coords.): Mujeres que gobiernan municipios. Experiencias, aportes y retos, México, COLMEX, 1998, pp. 13-27, y por Barrera, que alude al reconocimiento visual, histórico, social, político y cultural de las mujeres en la vida social.

2. El estado de Guerrero limita al norte con el estado de México y Morelos, al sur con el Océano Pacífico, al este con Puebla y Oaxaca y al oeste con Michoacán. Cuenta con una extensión de 
perdido en casi todos los casos el derecho a bosques, playas, tierras cultivables, etc. Además, el desarrollo, especialmente el turístico, ha beneficiado a capitales ajenos a la entidad o de no ser así a la clase caciquil local, sin ningún compromiso social con los habitantes. No puede olvidarse tampoco la larga lista de denuncias ecológicas presentadas, pues el medio natural ha sido transformado agresivamente en pro de la construcción de complejos turísticos que ha traído consigo la contaminación creciente de nichos ecológicos.

Cabe mencionar también el desplazamiento de los habitantes y la ocupación de las tierras por proyectos turísticos, proceso que da lugar a la principal problemática de carácter agrario en el estado, ya que estos proyectos se desarrollan muchas veces sobre tierras ejidales. Esto es resultado de un proceso que se identifica con el periodo coyuntural del Reparto Agrario en Guerrero y la instauración del modelo neoliberal, que incentivó el turismo y la explotación agroforestal, lo que después daría pie a la conformación de la nueva burguesía guerrerense.

Por su parte, Alba Teresa Estrada considera que la geografía del estado es decisiva en las formas del ejercicio del poder y la propia historia, comparando la Sierra Madre del Sur como un fuerte natural. Ya que, "por su difícil acceso y por el aislamiento de sus poblaciones, Guerrero ha sido a lo largo de su historia escenario bélico: desde la guerrilla insurgente en la Independencia hasta la guerrilla campesina de los años sesenta... la configuración misma del poder local, ha sido también, sin duda, propiciada por el aislamiento de la población»${ }^{3}$.

La división del territorio guerrerense en siete regiones, que corresponde a criterios geográficos, económicos, sociales, políticos y culturales, es pertinente de mencionar, ya que las políticas públicas ejercidas desde el nivel del gobierno estatal son aplicadas bajo esta división, lo cual conduce a cierta marginación en cuanto a la distribución de recursos estatales por regiones, sobre todo aquellas que presentan mayor población indígena o aquellas que no representan un beneficio comercial para el gobierno estatal o, en su defecto, concentran parte de los recursos destinados a una región sólo en algunos centros poblacionales, principalmente comerciales.

Gisela Espinosa y Miguel Meza apuntan:

«Es un estado pobre, el tercero más pobre del país, su economía y sus índices de bienestar social así lo indican. En 1990 Guerrero era la entidad, después de Zacatecas, con la tasa más alta de desempleo abierto y con menor proporción de población económicamente activa, respecto a la población total. El sector más desarrollado de su economía es el terciario, en él se ubica el 49\% de la PEA estatal, mientras el sector

$64.282 \mathrm{~km}^{2}$ y un litoral de $500 \mathrm{~km}$; esta superficie representa el 3,3\% del territorio nacional y ocupa el decimocuarto lugar entre las 32 entidades del país. Lo integran siete regiones: Zona Norte, Tierra Caliente, Centro, La Montaña, Acapulco, Costa Grande y Costa Chica. Los centros urbanos representativos de cada región son Taxco, de la Zona Norte; Ciudad Altamirano, de Tierra Caliente; Chilpancingo, del Centro; Tlapa, de la Montaña; Zihuatanejo, de la Costa Grande y San Marcos, de la Costa Chica.

3. Estrada Castañón, Alba Teresa: Guerrero: sociedad, economía, política y cultura, México, CIIH/ UNAM, 1994, p. 12. 
secundario ocupa apenas un $16 \%$ de la población trabajadora y el primario un $35 \%$. El $11 \%$ de la población son indígenas: nahuas, tlapanecos, amuzgos y mixtecos» ${ }^{4}$.

«Guerrero es, después de Chiapas, la entidad federativa con mayor porcentaje de monolingües $(28,9 \%) »^{5}$.

Estos datos valen para reconocer las difíciles condiciones económicas en las que vive la población guerrerense: falta de capacitación, de empleo, de integración en el modelo económico estatal, al cual acceden solamente como trabajadores de la industria turística; por algo el sector terciario llega a ser un 49\%, casi la mitad de la aportación económica estatal, lo cual refleja el rápido y casi depredador desarrollo de la industria turística ${ }^{6}$. A diferencia del desplome del porcentaje del sector primario, que es consecuencia directa de la falta de apoyos, proyectos y planificación de las actividades agrícolas por parte del Estado, además de ser una actividad que va en contra del actual modelo económico guerrerense. La situación resultante de ello es una gran oferta de mano de obra, principalmente rural e indígena, la cual migra a estados del norte del país, como Sinaloa, o al sur de Estados Unidos.

Aún cuando la agricultura ha dejado de ser la principal actividad, Guerrero todavía es un estado campesino.

«La agricultura de subsistencia predomina en el agro guerrerense. En Guerrero la unidad de producción agropecuaria fundamental es el ejido (...) ejidos y comunidades agrarias concentran en conjunto casi $60 \%$ de la superficie total del estado (...) los cultivos principales son el maíz, el cocotero, el café y el ajonjolí, que aportan más del $80 \%$ de la superficie total sembrada y cosechada y más del $70 \%$ del valor de la producción»".

\section{LOS AMUZGOS}

El origen de este grupo indígena se desconoce, pero investigaciones realizadas por parte de lingüistas encuentran en su idioma semejanzas con el mixteco, lo cual indica, según la teoría de Sahagún y Torquemada, que emigraron del norte junto con aquel grupo, siguiendo la ruta del Pánuco. De este modo, ascendieron a la Mesa Central, estableciéndose en Tula; después se vieron obligados a pasar a Cholula y más tarde al sur, al territorio del hoy estado de Oaxaca. Existen incluso hipótesis como la de Gay y Burgoa en la que se señala que emigraron de Sudamérica ${ }^{8}$.

A través de la historia oral y en el registro de la lengua amuzga, se asegura que tienen su origen en el mar, en algunas islas o "las tierras de en medio» (ndyuaa

4. Espinosa, Gisela y MezA, Miguel: "Guerrero en cifras: las dimensiones de la pobreza», en Armando Bartra (coord.): Crónicas del Sur. Utopias campesinas en Guerrero, México, Era, 2000, pp. 77-78.

5. Estrada Castañón, Alba Teresa: Op.cit., p. 9.

6. «Acapulco es la segunda ciudad que más creció en el país en la década pasada» (Ibíd., p. 10).

7. Ibíd., pp. 20-21.

8. GutiérRez Avila, Miguel Angel: Déspotas y caciques. Una antropología politica de los amuzgos de Guerrero, México, UAG, 2001, p. 65. 
xenncue) y que de ellas llegaron a la zona costera del pacífico a la altura donde están establecidos ahora los límites de los estados de Guerrero y Oaxaca.

Los amuzgos llaman a su lengua Nomnda, palabra compuesta que viene de las palabras Noom, que significa palabra o idioma y Ndaa, que quiere decir agua, o sea "Idioma o palabra del agua».Y ellos mismos se llaman $N_{n}$ 'anncue, que viene de las palabras $N n^{\prime} a n$, y quiere decir "personas» y Ncue, que quiere decir «de en medio».

La versión de que los amuzgos tienen su origen en el mar, o sea que vienen de las "Tierras de en medio", y que su idioma viene "del agua» adquiere un sentido lógico. Sin embargo, cabe señalar que la asignación de amuzgos (amoxco) fue una designación que alude a lo que éstos representaban para el imperio mexica. Por tanto, la traducción de este término como "lugar de libros» está relacionada con la función que cumplía el espacio que habitaban como centro administrativo, ya que los códices y documentos comunitarios siempre estuvieron bajo el cargo de las clases sacerdotales sin que tuviera acceso la clase popular.

La versión oral de la llegada de los amuzgos a Xochistlahuaca cuenta que habitaban las tierras cercanas a la costa del Pacífico, pero su cercanía con otros grupos étnicos de la zona de Oaxaca -que bien pudieron ser los mixtecos- provocó una migración a otros territorios para buscar establecerse lejos de conflictos interétnicos:

«Pensaron que era mejor irse de ahí, los principales hablaron y dijeron que era bueno buscar otras tierras donde los amuzgos pudieran reproducirse en paz. Tomaron unas piedras que eran como imanes y cuando llegaron a Xochistlahuaca las enterraron para que todos permanecieran unidos a esa tierra»?.

Otra interpretación, y aventurando una hipótesis, puede sugerir que los amuzgos son un grupo de tradición campesina y la constante alusión al elemento líquido es la manera de expresar su necesidad de éste para la supervivencia y el logro óptimo de la reproducción agrícola y con ella de la vida. Una referencia concreta la encontramos en la fiesta de San Marcos, en que los amuzgos realizan sacrificios de gallinas sobre piedras que míticamente representan a los truenos y rayos, que se supone es un rito de petición de lluvias, ya que se realiza en fechas previas a la preparación del cultivo. Por tanto resulta una cuestión abierta el conocimiento que los amuzgos tienen acerca de su propio origen.

\section{EL MUNICIPIO DE XOCHISTLAHUACA}

Este municipio está clasificado con un grado de marginación muy alta por el Centro de Desarrollo Municipal. Considerado como el municipio en cuarta posición de marginalidad en el estado de Guerrero, decimosexto en México, ello supone que Xochistlahuaca es uno de los municipios indígenas de Guerrero y México con mayor grado de problemática social, económica y de acceso a los

9. Entrevista a Agapito Valtierra, originario de Xochistlahuaca, quien fue Comisariado Ejidal. Noviembre de 1999. 
servicios básicos. Como otros municipios indígenas del país, Xochistlahuaca vive en condiciones de muy alta marginalidad, porque así lo ha colocado el proyecto de nación mexicana, proyecto en el cual la desindianización sigue estando presente. Una manera de lograrlo es reducir al mínimo las condiciones de vida de los pueblos indígenas. De esta manera las poblaciones indígenas no tienen cabida con propuestas propias, equitativas y correspondientes a su condición cultural (como la lucha por el territorio, concebido de manera diferente respecto a la cultura occidental por ejemplo).

Actualmente en Xochistlahuaca se identifica la presencia de dos poderes: el de las autoridades tradicionales y el del presidente municipal priísta, quien administra desde su casa los asuntos municipales. Mientras tanto en Xochistlahuaca la creación de organizaciones civiles como el Frente Cívico Indígena de Xochistlahuaca y Nación Amuzga están consolidando un nuevo ciclo político. Es relevante el hecho de la refuncionalización de las autoridades tradicionales, quienes se plantean la autonomía del pueblo, pero habría que revisar este proceso más de cerca, ya que se trata de una estructura jerárquica que también tiene sus propios filtros de acceso al poder, que por cierto ha sido restringido a las mujeres. También habría que plantearse si ignorar a los partidos políticos y los procesos electorales es una posible solución a los conflictos políticos, ya que de igual manera seguirán actuando en ese municipio.

\section{PERFILES DE LIDERESAS INDÍGENAS}

Una tarea que considero pendiente es el estudio de los perfiles de las lideresas y del contexto en el cual están participando, con el objetivo de conocer las condiciones, causas y consecuencias del desarrollo de los liderazgos femeninos, como metodología para descubrir cómo las mujeres acceden a la toma de decisiones y con ello al poder. Barrera indica, respecto a la importancia de analizar los liderazgos femeninos en la comunidad, que

«esperamos contribuir así a abrir camino a posteriores investigaciones en torno al perfil de las mujeres que gobiernan municipios en nuestro país, partiendo de la hipótesis de que el acceso de estas mujeres al poder municipal se basa en el arraigo y liderazgo ejercido por ellas en la comunidad, lo que permite sean lanzadas como candidatas de algún partido» ${ }^{10}$.

El caso de la participación de las mujeres indígenas en su municipio entrelaza y sobrepone varias situaciones que hacen de este hecho un fenómeno muy complejo: el alto grado de analfabetismo, monolingüismo, desnutrición y la cantidad de horas y cargas de trabajo, hacen que a las mujeres indígenas les resulte más difícil su acceso a la participación social y política y a los espacios de poder, lo cual no ha imposibilitado sin embargo su fundamental y numerosa participación en las movilizaciones políticas.

10. BarRera Bassols, Dalia: «Mujeres que gobiernan municipios: un perfil», en Dalia Barrera Bassols y Alejandra Massolo (coords.): Op. cit., p. 9. 
Actualmente la presencia de mujeres en cargos públicos en municipios indígenas representa una minoría dentro de la población marginada en la estructura de poder nacional, sin embargo esta participación es sumamente significativa, puesto que no sólo ocupa lugares negados, sino que puede arrojar mucha luz sobre cómo acceden y ejercen el poder las mujeres indígenas, cómo se organizan, cómo y través de qué ejercen la ciudadanía, cómo y por qué votan, etc. Lo cual sugiere que una de las tareas urgentes para los investigadores y las investigadoras es la realización de perfiles de las mujeres indígenas, desde aquellas que "mandan», hasta las que participan desde la base. Todo ello con la intención de comprender mejor el proceso histórico, social y cultural de la participación de las mujeres indígenas en los movimientos, en los partidos y en sus comunidades, porque aún hoy sabemos muy poco al respecto.

De ahí la importancia de indagar y hacer visibles los pocos casos que conocemos. En esta comunidad, las mujeres tienen una presencia importante en las movilizaciones políticas, así como en los procesos electorales, sin embargo son poco reconocidas o no acceden a la toma de decisiones en los propios movimientos políticos en los que participan. Los ejemplos aquí presentados son únicos y éste es el principal criterio de elección para la realización de entrevistas, las cuales corresponden a un guión que bajo el formato de pregunta-respuesta abarcó el análisis del origen familiar, el acceso a la educación, la infancia, la adolescencia, la inserción en actividades sociales, que por ende condujeron a una inclusión en el ámbito público local y en la vida política de la comunidad. Se muestra un especial interés en los roles de género que han marcado y han sido interiorizados por estas mujeres, definiendo su trayectoria de lideresas.

En primer lugar la observación y la observación participante fueron técnicas utilizadas, al ser consideradas fundamentales para el trabajo etnográfico y antropológico. Estas entrevistas se realizaron a profundidad y fueron utilizadas para obtener información central del ciclo de vida, desde los distintos niveles sociales, culturales, económicos y de género de las lideresas. Se trabajó también con información complementaria de entrevistas aplicadas a otras mujeres de la comunidad.

Siguieron la sistematización de datos que la metodología de las historias de vida ofrece. Se aplicó esta técnica de análisis a las tres mujeres que representan un liderazgo formal o informal de las mujeres de Xochistlahuaca: Florentina López de Jesús, Josefina Flores y Martha Sánchez Néstor. Con este método se buscó sistematizar la información obtenida en las entrevistas. Junto con ello, el análisis de los contextos históricos de la mujer, el perfil de la lideresa o activista política. A través de la investigación de la historia de vida de cada lideresa, se logró ubicar información representativa. Es decir se ha elegido a estas tres mujeres porque cada una representa el ejemplo o el "caso» de la actividad o la participación de las mujeres en esa generación.

Se acotó un periodo histórico de 80 años aproximadamente, desde la aparición pública de la primera lideresa hasta la más reciente. Todo ello con la intención de conocer y reconocer la trayectoria de cada una de estas mujeres indígenas y así ubicar la importancia y su difícil conformación como lideresas, 
debido principalmente a las condiciones sociales, políticas y culturales de la región pero en especial por su condición de mujeres.

\section{FORMAS DE PARTICIPACIÓN DE LAS MUJERES EN LAS COMUNIDADES INDÍGENAS}

En el caso de las mujeres indígenas políticamente activas es imprescindible analizar las condiciones de participación, su forma y contenido, para conocer más sobre este tipo de participación y sobre ellas mismas como sujetos sociales. Paloma Bonfil registra que:

«En términos generales, la participación de las mujeres indígenas se ha dado también en dos niveles: en los espacios y las formas que tradicionalmente les han sido accesibles dentro de sus comunidades y que, con la nueva forma y circunstancia de su participación, se han politizado; y a través de organizaciones no tradicionales que ellas han conformado como un medio para lograr fines concretos (satisfactores económicos, servicios, recursos culturales); y como un factor de toma de conciencia étnica y de género y para la colocación de sus demandas particulares en las agendas más amplias de sus pueblos» ${ }^{11}$.

Partiendo de lo anterior identificamos dos formas de participación en las mujeres indígenas: la tradicional y la no tradicional. La primera remitirá generalmente a oficios tradicionales asignados a las mujeres de la comunidad: tejedoras, alfareras, tortilleras, parteras, bordadoras, panaderas y, en el caso de algunas regiones y comunidades rurales, la elaboración de comida o la venta en los mercados regionales de productos propios o no. Estos oficios primero han pasado por un proceso de supervivencia a las condiciones sociales, económicas y culturales, debido a que pudieran parecer obsoletos, pero para la comunidad siguen siendo de subsistencia y comportan una estrategia de ahorro, al evitar la adquisición de productos que tendrían que comprar en los centros mestizos (como los vestidos o el pago de servicios médicos, que implica un traslado y eleva el costo). Culturalmente, representan la práctica de seguir realizando los oficios tradicionales, que contribuye a conformar la identidad del grupo étnico. Ello origina un proceso de resignificación, por el que los habitantes valoran lo realizado artesanalmente, y contribuye a un sentimiento de orgullo de lo "propio".

En el caso de la participación en las organizaciones no tradicionales, cabe señalar que han atravesado un proceso histórico y de selección. El ámbito docente puede ser un primer campo de participación de las mujeres indígenas, aunque altamente limitado, ya que representa, en primer lugar, acceso y producción de conocimiento y, en segundo lugar, acceso a la palabra, cuestiones tradicionalmente negadas a las mujeres.

11. BonflL, Paloma: «Las mujeres indígenas y su participación política: un movimiento contra la desmemoria y la injusticia», en Dalia Barrera Bassols (comp..): Participación política de las mujeres y gobiernos locales en México, México, GIMTRAP, 2002, p. 71. 
De otra parte, los ejemplos más inmediatos de las organizaciones no tradicionales de mujeres indígenas aluden a una serie de programas aplicados a través de políticas públicas nacionales e internacionales (como el Banco Mundial) dirigidas a mujeres campesinas e indígenas (Mujeres en el desarrollo rural de la Secretaría de Agricultura, Ganadería y Pesca o Mujer campesina de la Secretaría de la Reforma Agraria), que en su mayoría están diseñados como programas de combate a la pobreza, sin una verdadera perspectiva de género, en donde las mujeres sean vehículos para incrementar los índices de bienestar social.

Otro ejemplo de la participación en organizaciones no tradicionales lo representan todas aquellas asociaciones que demandan derechos de las comunidades y pueblos indígenas, en las cuales están inscritas algunas mujeres indígenas y que están relacionadas con el acontecer político e histórico, en el cual los hombres, las mujeres y las comunidades indígenas están involucrados.

En el caso de Xochistlahuaca, he identificado ambas vías de participación femenina. Respecto a la organización tradicional, los grupos de mujeres artesanas (tejedoras de telar de cintura) representan una fuerza política en la comunidad y un espacio de acción, opinión y relativa autonomía de las mujeres. En cuanto a la organización no tradicional, las maestras de la comunidad son activas participantes en política, interesadas en las decisiones que afectan o benefician a su comunidad y han sostenido desde la base y con diversas movilizaciones el actual Frente Cívico Indígena de Xochistlahuaca, movimiento de maestros que ha tomado desde enero de 2001 el Palacio Municipal de Xochistlahuaca y que después de una fuerte presión lograron que la Presidenta Municipal Aceadeth Rocha solicitara licencia al Congreso local y abandonara la comunidad, tras un enfrentamiento entre maestros y un grupo de choque que apoyaba a la Presidenta Municipal.

En ambas formas de participación, dentro de las comunidades indígenas, cabe la reflexión de los patrones específicos culturales asignados a hombres y mujeres, niños, jóvenes, adultos y ancianos de cada etnia y es posible que de cada comunidad, ya que considero que existen diferentes grados de aceptación o prohibición de la incursión de las mujeres indígenas en lo público, de acuerdo con el grupo étnico, la región, etc. Es importante la presente observación, ya que incidirá en que la comunidad considere en menor o mayor grado a una lideresa como una transgresora. Esto, partiendo de que existe una estructura política indígena que rige las comunidades indígenas más o menos similar, en donde el parentesco y los grados jerárquicos están marcando el acceso de los hombres de la comunidad a los cargos socio-políticos.

La participación de las mujeres indígenas, por todo lo mencionado anteriormente, es peculiar y difícil. Tiene sus propios filtros de selección, como la disponibilidad de tiempo, la pertenencia a una familia determinada, el propio proceso de empoderamiento colectivo y/o individual, en el caso de las lideresas, por el que han pasado las mujeres. Por ello Paloma Bonfil considera que:

«...aún los nichos disponibles y los disputados para la participación pública y política de las mujeres se encuentr[a]n en distintos grados de accesibilidad para las mujeres de una misma comunidad. Efectivamente, las posibilidades y la inciden- 
cia de la participación femenina en la vida pública y en el control del poder y la autoridad dentro de las comunidades indígenas y sus mecanismos de decisión son diferenciadas en función de la posición y condición de esas mismas mujeres, a su vez determinadas por su nivel socioeconómico, su estatus marital, el prestigio del cónyuge o sus antecedentes familiares» ${ }^{12}$.

La participación pública de las mujeres indígenas está siempre mediada por las condiciones de género, es decir, expresamente el sistema tradicional ha negado el acceso de las mujeres al espacio público, lo cual es reforzado por las normas que regulan el ingreso en la estructura política tradicional, tales como que se debe ser varón, padre de familia y comunero. Además existen otros candados menos visibles que hacen que las mujeres indígenas limiten su participación política. Estos filtros de control son: la disponibilidad de tiempo, la desinformación general (esto precisamente por no asistir a las asambleas, por ejemplo), los sentimientos de miedo, temor, vergüenza, inseguridad, el desprestigio como consecuencia de participar en lo público y los celos que en la comunidad se despiertan por quienes acceden al poder. Por ello es importante conocer y reconocer las historias de vida de lideresas amuzgas o de mujeres que han logrado acceder a puestos de poder en Xochistlahuaca, para así ubicar cómo operaron estos mecanismos y cuáles fueron las estrategias para la superación de los obstáculos. A partir de testimonios de mujeres que han participado políticamente, se ha identificado lo siguiente en cuanto a las limitaciones de las lideresas (indígenas y mestizas) en el acceso a los espacios de poder:

«En las instituciones y espacios como los sindicatos, partidos políticos, movimientos sociales, comunidades rurales e indígenas, existen ciertas actitudes permanentes como la oposición y el bloqueo hacia las mujeres por parte de otros miembros del movimiento o partido, femeninos o masculinos, así como dudar de su capacidad de mando y dirigencia, o su segregación de ciertas tareas, invisibilidad de las iniciativas femeninas, segregación y discriminación de las mujeres de los espacios políticos y de poder, considerados como "masculinos»; funcionan de manera contundente reglas no escritas que imponen la subordinación de las reivindicaciones de las mujeres. Paradójicamente, del rol de madre-esposa surgen las motivaciones más generalizadas para la participación social y política de las mujeres (ante la imposibilidad de cumplirlos cabalmente); pero también surgen de ahí los costos de la participación: culpa, estrés, ansiedad por "descuidar» dicho papel y sus requerimientos, violencia intradoméstica, celos, presión, etc.» ${ }^{13}$.

El salir de casa a espacios y ocupaciones no permitidos o legítimos para las mujeres será castigado mediante diversas formas de violencia (amenazas, chantajes, insultos, chismes, maledicencias, etc. $)^{14}$. Así entendemos que el poder en

12. BonflL, Paloma: Op. cit., p. 74.

13. Barrera Bassols, Dalia y Aguirre Pérez, Irma: Participación política de las mujeres. La experiencia de México, México, CONACULTA/INAH, 2002, pp. 18-19 y 29.

14. BARRERA BASSOLS, Dalia: "Transgresiones, control social y violencia hacia las mujeres en el ámbito rural». Ponencia presentada en el 3er. Congreso «Los actores sociales frente al desarrollo rural» de la Asociación Mexicana de Estudios Rurales, Zacatecas, México, junio de 2001, p. 2. 
el interior de las comunidades indígenas se sigue considerando un asunto principalmente masculino, en donde se superponen el sistema de género que dicta un tipo ideal de ser mujer (quien es elegida como esposa cambia su residencia y pertenencia por el grupo del esposo, tiene como principal función social y cultural la maternidad, dedica su tiempo a todas las actividades relacionadas con ese rol de madres y mujeres de un varón, etc.) y los sistemas de poder que la comunidad tiene establecidos en donde sólo los varones tienen derecho a heredar tierra del grupo familiar y ello los hace personas con derecho participar en las asambleas ${ }^{15}$, proporcionándoles por lo tanto ciudadanía. A su vez los varones tienen derecho a escalar en la jerarquía del sistema de cargos cívico-religiosos que es fundamental en la organización indígena para colocar social, económica y políticamente a los individuos de la comunidad, proporcionándoles estatus y poder.

Las mujeres sólo aparecen en este sistema de cargos como acompañantes de sus esposos, y alejadas de detentar ese poder; además están limitadas por factores que obstaculizan su participación como anteriormente se ha mencionado (sólo hablar la lengua materna y no ser bilingües, no disponer de tiempo, el efecto de las maledicencias sobre una mujer interesada en los asuntos públicos, la desinformación e incluso en algunos casos la negación del acceso a la información por parte de los varones de la comunidad y de los propios ejecutores de políticas públicas que no pertenecen a la comunidad pero que de igual forma valoran inferior el interés o las actividades de las mujeres, etc.) y por reglas que el sistema de Usos y Costumbres $^{16}$ dicta, como aquella que considera el espacio doméstico el lugar «natural» e idóneo de las mujeres, por ejemplo.

Cabe mencionar que en esta relación de poder-sistemas de género la educación escolarizada admitida hasta hace poco (a partir de los años sesenta en la comunidad de Xochistlahuaca), y a la que sólo asistían los niños en un principio, ha proporcionado sin duda herramientas de participación y acceso a la vida pública a las mujeres, por lo que ha influido para la reconfiguración de los procesos de poder-género, lo que no evita que las mujeres activistas tengan que enfrentar obstáculos que tienen que ver con su condición de mujer. Ello es lo que hace tan valioso el caso de las trayectorias de las lideresas que en este artículo se mencionan.

En el caso de las mujeres de Xochistlahuaca se presentan todos estos elementos, pero también hay quienes han logrado rebasar esa barrera cultural. Existe el caso de la lideresa tradicional Florentina López de Jesús, tejedora de telar de cintura y Presidenta de la Cooperativa artesanal «La flor de Xochistlahuaca", quien ha logrado participar, ocupar puestos de poder, obtener el reconocimiento interno y externo y la participación en la toma de decisiones en

15. Las asambleas siguen siendo el principal órgano que legitima la toma de decisiones comunitarias.

16. El sistema de Usos y Costumbres es la normatividad no escrita que regula la vida social y cultural de las comunidades indígenas y establece qué está bien y qué está mal visto dentro de la comunidad; si se trasgrede puede ser motivo de castigo. 
la comunidad, a través del trabajo de gestión de recursos para la elaboración y venta de textiles amuzgos, siendo reconocida internacionalmente como una de las principales expositoras del Arte Popular Mexicano ${ }^{17}$. Contamos con el caso de Josefina Flores García, maestra bilingüe que en 1977 y a la edad de 23 años fue la primera Presidenta Municipal de Xochistlahuaca, y de quien resulta muy significativo en su historia personal el acceso al conocimiento y a la información política regional desde su puesto de directora de escuela primaria ${ }^{18}$.

Cabe destacar el caso de la lideresa Martha Sánchez de 30 años de edad, que inicia su trayectoria política como secretaria de una de las organizaciones sociales y políticas más importantes de este estado, «Consejo Guerrerense 500 años de resistencia indígena y negra». Su primera obligación fue la defensa de la marcha de indígenas guerrerenses «No están solos» en apoyo al Ejercito Zapatista Liberación Nacional, que salió de Chilpancingo a la ciudad de México.

«La marcha me permitió a mí, porque empiezo a ver gente de Xochis, gente de acá, o sea yo no había entendido hasta ese momento realmente qué hacía el Consejo, lo entiendo en el tramo y viniendo en la marcha, pasando por los pueblos, escuchando los discursos de los hombres. Había ahí una señora de la Montaña, dentro de la dirigencia, pero que ella estaba así, que nadie la tomaba en cuenta y ella se bronqueaba fuerte con ellos, pero ahí las banderas las llevaban los otros, los otros compañeros. Me empiezo a involucrar y me dicen "llévate tu máquina», pero yo siento que ese momento, me llevo la máquina y yo hacía todo el rollo técnico, que haz este cuadro, que el otro, pero siento que me voy identificando con esa lucha en el tramo, todavía no me había comprometido siento, pero me voy identificando y digo 'a mí me gusta hacer esto'» 19 .

Martha Sánchez aparece como un liderazgo representativo de lo que ha venido sucediendo con la participación política de las mujeres indígenas, las lideresas indígenas. Muchas son jóvenes, hablantes de español, con un grado de escolaridad de secundaria por lo menos y activas gestoras de los intereses prácticos y estratégicos de las mujeres de su comunidad. De otra parte, esta generación de nuevas lideresas indígenas ha tenido acceso a otro tipo de información y ha sido influenciadas, por un lado, por el movimiento indígena, en uno de sus momentos históricos más importantes, en el que junto con la presencia del Ejécito Zapatista de Liberación Nacional, la discusión sobre la cuestión étnica no sólo ha rebasado los límites locales y regionales, sino hasta los nacionales, volviéndose una causa internacional; y por otro lado, por el movimiento feminista, que parece haber logrado llevar el tema de las mujeres a la agenda de temas de interés internacional, nacional y local.

Estas nuevas líderes indígenas innovadoras están trabajando por superar las condiciones de marginación y de pobreza, pero también están preocupadas por transformar y cambiar todas aquellas situaciones sociales y culturales que reproduzcan la subordinación de las mujeres en la vida de la comunidad.

17. Entrevista a Florentina López de Jesús, 21 de abril del 2001.

18. Entrevista a Josefina Flores García, 24 de agosto de 2001.

19. Entrevista a Martha Sánchez Néstor, 1 de febrero de 2002. 
Existe una constante lucha y activismo por la construcción de la ciudadanía, el conocimiento y el ejercicio de los derechos humanos, los derechos sexuales y de las mujeres, cuestión profundamente política. Hasta el derecho al amor se ha vuelto conciencia en la construcción de estas nuevas identidades, que están redimensionando la realidad de las mujeres y los hombres indígenas. Su interés por lo público y por el acceso a la toma de decisiones en el ámbito local y/o municipal está implicando nuevas situaciones.

Martha Sánchez es también representativa en lo que respecta a la región. Ella como otras amuzgas han tenido que emigrar de Xochistlahuaca en búsqueda de alternativas de supervivencia. Las amuzgas con mayor índice de pobreza emigran temporalmente como pizcadoras de tomate a Sinaloa; la mayoría se traslada a regiones dentro del mismo estado como trabajadoras domésticas y una minoría se va con deseos de capacitarse y estudiar. La remuneración por el trabajo doméstico es la alternativa de muchas amuzgas, se ha convertido en una estrategia en la que se usa la mano de obra de las mujeres de la familia, quienes a veces ni siquiera deciden por ellas mismas esta salida de la comunidad.

«Bueno, me iba con mi prima, su sobrina pues de mi mamá; me voy a Iguala con ella, llego a esa casa, o sea para mí una gente desconocida, un mundo desconocido, llego y me inscribo. Yo ahí, un sueño que me ha perseguido toda la vida ha sido eso, yo ahí hacía el aseo, tendía las camas, no lavaba, no planchaba, la lavada le tocaba a mi prima, la planchada ella también planchaba, yo hacía el aseo barría el garaje, lavaba los trastes, hacía el almuerzo a la maestra, hacía la comida, me tocaba a mí, agua fresca. Yo llegaba a una casa que no era como mi casa pues, ahí se cocinaba diario sopa, la comida fuerte y el postre, así, o sea ahí me empiezan a meter a ese rollo de la guisada. Algo que me chocaba, que me traumaba era que la maestra decía «con aceite rojo -primera vez que conocí el aceite rojo- se limpian estos muebles» los coloniales, hasta que relumbraran ${ }^{20}$.

Martha Sánchez vivió la experiencia de la emigración para realizar un trabajo doméstico remunerado, como trabajadora doméstica en Iguala en el estado de Guerrero. Ahora su actividad social y política la obliga a residir en Chilpancingo, porque como lideresa en Guerrero tiene que estar al tanto de la información y los acontecimientos sociales y políticos, porque de otra manera la información no llega hasta la región amuzga y, si llega, pasará por los filtros de control caciquil, por lo que ella siempre está en constantes viajes entre la zona amuzga y el centro del estado. Esta situación de movilidad intensiva es también una característica de los nuevos liderazgos femeninos indígenas, ya que su activismo político no se limita ni a su región, ni a la etnia a la que se pertenece. La relación con otras mujeres indígenas es una situación más o menos consolidada en el estado de Guerrero, es consecuencia del propio desarrollo del movimiento indígena. Ello les ha permitido reflexionar y socializar problemáticas comunes.

20. Ibíd. 
De otra parte, la emigración es un fenómeno que trae consigo sus aspectos favorables; es decir las mujeres emigrantes al enfrentarse a nuevas situaciones desarrollan habilidades, al tener acceso a un trabajo y un ingreso (que en el mejor de los casos podrán gestionar en menor medida) pueden como consecuencia desarrollar un proceso de empoderamiento personal. Este es el caso de Martha Sánchez.

Al vivir de cerca la violencia y el ejercicio del control de la población, con Figueroa Alcocer ${ }^{21}$, Martha Sánchez (como Florentina López) se enfrentó al principal cacique de Guerrero, expresándose así lo que es ser mujer e indígena y vivir en Guerrero.

Martha Sánchez tiene como objetivo la creación y el acceso a los recursos económicos y de capacitación para las mujeres indígenas guerrerenses. En Xochistlahuaca ha organizado ya grupos de artesanas; pero consciente de que resulta insuficiente para una mejor calidad de vida de las mujeres indígenas el sólo responder a los intereses prácticos para mejorar la condición de las mujeres, esta lideresa junto con otras lideresas indígenas está revolucionando sus comunidades y la vida de las mujeres, al promover e introducirse en actividades como talleres de derechos humanos, derechos de la mujer, realización de diagnósticos sobre mortalidad materna junto con organizaciones civiles de cobertura nacional, etc., actividades que responden a intereses estratégicos, con el objetivo de modificar la posición de las mujeres indígenas en el interior de sus comunidades. Ello sin duda está repercutiendo en el reacomodo de las fuerzas, no sólo frente a los hombres, sino frente a las propias mujeres.

Finalmente, esta lideresa considera que un ámbito de acción es el espacio municipal y considera que cuando se logre la transparencia en los procesos electorales del municipio, se podrá eliminar el clientelismo, mecanismo que ha sostenido al PRI y al sistema de caciques en la región, del cual las mujeres indígenas en general y las amuzgas en ese municipio son uno de los principales sectores objetivo. Por estas consideraciones, cree que la incursión como candidata en los procesos electorales y la defensa del voto son una lucha que las mujeres indígenas también tienen que dar.

\section{CONCLUSIONES}

Las mujeres y especialmente las mujeres indígenas están fuertemente influidas en sus acciones, movilizaciones y deseos por la conciencia de lo comunitario, lo colectivo. Lo personal recién empieza a construirse con las peculiaridades que implica el pertenecer a un grupo étnico; las mujeres indígenas seguramente no llegarán al mismo concepto de lo individual que las mujeres no indias. El cuestionamiento para el caso de las mujeres indígenas es si lo colectivo debe ser parte de lo individual o si el colectivo debe permitir el derecho a la individualidad, para lo que aún no se tiene una respuesta. Una mujer indígena, en un foro, aseveró que ella «era primero indígena, después mujer

21. Gobernador Constitucional del Estado de Guerrero de 1993-1996. 
y luego mexicana» ${ }^{22}$, lo cual refleja el orden de prioridad en la construcción identitaria de las mujeres indígenas.

La participación política y el desarrollo de liderazgos de mujeres sin duda están propiciando una nueva etapa en las comunidades indígenas, donde las mujeres son agentes de cambio. En este caso, las lideresas de Xochistlahuaca han aportado información valiosísima que, dejando a un lado cualquier tendencia ideológica o política, dio cuenta de las formas y las vías de participación de las mujeres indígenas en el ámbito local, que eran mi principal interés. Sin embargo, al investigar estos tres casos, he encontrado diferencias, semejanzas y ciertas tendencias. Respecto a las diferencias, cabe indicar que son tres liderazgos dados en contextos y condiciones diferentes y desarrollados desde los distintos grupos sociales que representan a las mujeres de Xochistlahuaca. Incluso en los casos de Florentina López y Josefina Flores, quienes pertenecen al mismo partido, esa militancia resulta de carácter diferente y, aunque priístas, su liderazgo y su afiliación son de índole y origen distinto; por ejemplo, la primera se enfrentó al cacique de Xochistlahuaca, mientras que la segunda aparece como un instrumento del sistema de caciques y del partido. De igual manera, la primera se moviliza por las necesidades de las mujeres y siempre tiene presente el servicio a la comunidad, mientras que el compromiso de la segunda es con el partido y los grupos políticos locales.

Todo ello está determinado por el contexto generacional, cuestión que se observa de manera más clara en el caso de Martha Sánchez, que ha desarrollado su liderazgo a partir de los tiempos propios de la comunidad, pero en interacción con los tiempos ajenos a ella. Esta conciencia de ir y venir entre lo local, lo regional, lo nacional y hasta lo internacional está en gran medida influenciado por la generación, de ahí que Martha sea una lideresa joven, con preparación, estrategias discursivas y con presencia en el movimiento de mujeres indígenas. Este testimonio aporta también información sobre el desarrollo del movimiento indígena y en especial sobre el caso específico de la organización de las mujeres indígenas en Guerrero, proceso que se ha iniciado recientemente.

A esto tendría que agregar que el momento histórico ha definido e influenciado el tipo de participación y liderazgo en el que estas mujeres empezaron a desempeñar su liderazgo, aunque también es cierto que existen aptitudes y actitudes características personales para que lideresas y tiempo histórico coincidan.

Una de las semejanzas identificadas en estos casos es que si bien estas tres lideresas han vivido en condiciones de pobreza y marginalidad, no pertenecen al sector de mayor pobreza dentro de la misma comunidad, lo cual confirma una tendencia propia de las lideresas indígenas, por la cual las mujeres que acceden a espacios de poder poseen ciertas ventajas sobre otras mujeres, como un ma-

22. Hermelinda Tiburcio, mixteca de Rancho Nuevo de la Democracia, Gro, en el Foro «Voces de las mujeres indígenas de Guerrero", realizado el 7 de marzo de 2003, en Chilpancingo Gro. 
yor nivel educativo, la pertenencia a una familia con tradición de participación y una relativa presencia social.

De esta manera, tipifico de manera referencial estos liderazgos de la siguiente manera: Florentina López surge como una lideresa desde el espacio femenino tradicional; Josefina Flores simboliza la transición en la elección de autoridades locales en asamblea, por elección del PRI, y desarrolla su liderazgo desde el espacio institucional, reafirmando la cultura de sistema caciquil local y regional; y Martha Sánchez es una lideresa representativa de los movimientos y acciones actuales de una gran mayoría de las mujeres indígenas pues encarna un liderazgo desarrollado y fortalecido dentro del nuevo movimiento de las mujeres indígenas, el feminismo indígena.

De otra parte, el municipio de Xochistlahuaca remite a situaciones similares que están atravesando las comunidades indígenas de México: el debilitamiento e incluso el desplazamiento del sistema tradicional de elección de autoridades locales por la elección de autoridades municipales a través de los partidos políticos es una de las problemáticas más importantes y fundamentales en el devenir y el futuro de las comunidades. Ésta es causa de movilizaciones, de enfrentamientos y violencia cotidiana. Sin embargo, aunque esta problemática aparece como la más evidente, tal vez por su carácter público, la discusión principal es el rumbo de las sociedades indígenas en el contexto actual.

La tensión está dada, primero en torno al derecho de los grupos étnicos a la decisión sobre sus comunidades $y$, en segundo lugar, en caso de que este derecho se considere válido, en el desencuentro entre el proyecto de vida de las comunidades y el proyecto nacional. A su vez, en el interior de las comunidades indígenas se están evaluando y reconsiderando las desigualdades, proceso en que las mujeres están jugando un papel fundamental, para que las desigualdades que han venido experimentando desde siempre se equilibren, para poder así formar un frente de lucha que defienda sus demandas como grupo étnico frente a la sociedad mestiza.

Por ello los ayuntamientos así como los partidos políticos están siendo piezas clave en la definición de este nuevo rumbo que las comunidades indígenas están decidiendo. Tanto los primeros como los segundos están considerados como espacio de poder de la cultura mestiza, pero también pueden ser transformados y volverse espacios de apropiación, como las autoridades tradicionales de Xochistlahuaca han sugerido con su acción de la toma del palacio municipal.

Asimismo los movimientos sociales locales están siendo ámbitos importantes de participación y acción política. El movimiento indígena ha sido un espacio destacado para indígenas e incluso mestizos. También el movimiento de las mujeres indígenas es un espacio de gran importancia en la actualidad, ya que está propiciando una profunda transformación de las propias actoras y de la sociedad, dando cabida a la reflexión, la expresión, la lucha, la búsqueda y la construcción de sentidas demandas más acordes con la realidad en la que viven las mujeres indígenas. 


\section{BIBLIOGRAFÍA}

BARrera Bassols Dalia: «Mujeres que gobiernan municipios: un perfil», en Dalia Barrera Bassols y Alejandra Massolo (coords.): Mujeres que gobiernan municipios. Experiencias, aportes y retos, México, COLMEX, 1998, pp. 91-112.

- : "Transgresiones, control social y violencia hacia las mujeres en el ámbito rural». Ponencia presentada en el Ber. Congreso "Los actores sociales frente al desarrollo rural» de la Asociación Mexicana de Estudios Rurales, Zacatecas, México, junio de 2001.

- y Agurre Pérez, Irma: Participación política de las mujeres. La experiencia de México, México, CONACULTA/INAH, 2002.

BonfIL, Paloma: «Las mujeres indígenas y su participación política: un movimiento contra la desmemoria y la injusticia», en Dalia Barrera Bassols (comp.): Participación política de las mujeres y gobiernos locales en México, México, GIMTRAP, 2002, pp. 67-98.

estrada Castañón, Alba Teresa: Guerrero: sociedad, economía, política y cultura, México, CIIH/UNAM, 1994.

Espinosa, Gisela y Meza, Miguel: "Guerrero en cifras: las dimensiones de la pobreza», en Armando Bartra (coord.): Crónicas del Sur. Utopias campesinas en Guerrero, México, Era, 2000, pp. 75-102.

Gutiérrez Avila, Miguel Ángel: Déspotas y caciques. Una antropología política de los amuzgos de Guerrero, México, UAG, 2001.

MAssolo, Alejandra: "Introducción. Gobierno municipal y mujeres: un encuentro posible», en Dalia Barrera Bassols y Alejandra Massolo (coords.): Mujeres que gobiernan municipios. Experiencias, aportes y retos, México, COLMEX, 1998, pp. 13-27. 